\title{
Protein secondary structure prediction by combining hidden Markov models and sliding window scores
}

\author{
Wei-Mou Zheng \\ Institute of Theoretical Physics, Academia Sinica, Beijing 100080, China, \\ and \\ Bejing Genomics Institute, Academia Sinica, Beijing 101300, China
}

\begin{abstract}
Instead of conformation states of single residues, refined conformation states of quintuplets are proposed to reflect conformation correlation. Simple hidden Markov models combining with sliding window scores are used for predicting secondary structure of a protein from its amino acid sequence. Since the length of protein conformation segments varies in a narrow range, we ignore the duration effect of the length distribution. The window scores for residues are a window version of the Chou-Fasman propensities estimated under an approximation of conditional independency. Different window widths are examined, and the optimal width is found to be 17 . A high accuracy about $70 \%$ is achieved.
\end{abstract}

PACS number(s): 87.10.+e,02.50.-r

\section{Introduction}

There is an increasing gap between knowledge of protein structure and protein sequence. A long-term goal of the protein-folding problem is to predict the folded 3D structure of a protein from its amino acid sequence alone. Secondary structure prediction is an initial starting point in predicting the structure of a protein. The first efforts to predict protein secondary structures begun in the early 1970s (Chou and Fasman, 1974a and b). Since then, a number of sophisticated methodologies, e.g. statistical methods based on information theory, nearest neighbor methods, hidden Markov models, and neural networks, have been developed. Many algorithms examine a sequence window of 13-17 residues, assuming that the central amino acid in the window will adopt a conformation that is determined by all the amino acid residues in the window.

Solovyev and Shindyalov (2002) classified prediction algorithms into three categories: methods using single sequences, combined methods using nearest neighbors, and those using homology information. Although the highest prediction accuracy is obtained with methods using homology information, the methods require a homology search of related sequences and often an additional multiple sequence alignment. While the combined methods using nearest neighbors directly compare the window to be predicted with a segment database, the methods using single sequence convert such a database to parameters, so at the prediction step no datasets are referred, and then least computation is required. Most window-based methods require discriminant thresholds and a post-prediction filtering. 
Hidden Markov models (HMMs) (Rabiner, 1989) have been applied to molecular biology, in particular in gene-finding. HMMs have also been used for protein structure studies (Asai et al.,1993; Stultz et al., 1993; Karplus et al., 1997; Camproux et al. 1999). A probabilistic approach similar to the gene finder Genscan has been developed for protein secondary structure prediction without using sliding windows (Schmidler, Liu and Brutlag, 2000). In terms of Bayesian segmentation, the method integrated explicit models for secondary structure classes helices, sheets and loops with other observed structure aspects such as segment capping signals and length distributions, and reached an accuracy comparable to the information theory approach GOR (Garnier, Osguthorpe, and Robson, 1978; Gibrat, Garnier, and Robson, 1987; Garnier, Gibrat, and Robson, 1996).

Compared with DNA sequences, protein sequences are generally short, and their amino acid alphabet is of a large size 20. The range of lengths of secondary structure segments is rather small. The effect of duration might play a less important role. Conformation states in most window-based predictions are single residue conformations: coils(c), extended strands(e) and helices(h). Correlation among residue conformations is not taken into account. Here we develop a simple hidden Markov model with correlation of residue conformation included for the secondary structure prediction. We consider quintuplets of residue conformations as new states for hidden Markov processes. We have proposed a scoring scheme for emission probabilities based on amino acid alphabet reduction (Zheng, 2003). We shall discuss a scoring scheme based on sliding windows. By combining sliding windows with HMMs, outcome from individual windows may be synthesized, and hence error of prediction be reduced.

\section{Methods}

As in most methods, we consider 3 states $\{h, e, c\}$ generated from the 8 states of Kabsch and Sander (1983) by the coarse-graining $H, G, I \rightarrow h, E \rightarrow e$ and $X, T, S, B \rightarrow c$. Let $R \equiv R_{1: n}=R_{1} R_{2} \ldots R_{n}$ be a sequence of $n$ amino acid residues, and its corresponding secondary structure sequence be $S=S_{1} S_{2} \ldots S_{n}$. The structure prediction is the mapping from $R$ to $S$.

\subsection{Hidden Markov processes}

Instead of single residue conformations $c, e$ and $h$, our states of the hidden Markov chains are quintuplets of residue conformations, or our model is of the fifth order. The main restriction to a structure sequence is that the shortest length of the consecutive state $h$ must be 3 , and that of $e$ be 2 . As a direct outcome of this constrain, only 75 of the total $3^{5}=243$ possible quintuplets are legitimate. Exclusion of 7 rare ones (eceeh, hceeh, heece, heech, heeeh, heehh, and hheeh) further reduces the total number of states into 68, which are listed in Table 1. The number of transition states from any states are at most three. The transition rates may be estimated from hexamer and quinmer string counts. For example, the rate

$$
T(h h c c c \rightarrow h c c c c)=n(h h c c c c) / n(h h c c c),
$$

where $n(x)$ denote the count of string $x$.

\subsection{Emission probabilities}

Emission probabilities relate residue sequences to structure sequences. For the purpose of inferring the conformation of the central residue from a window flanking the residue, a form of emission probabilities is 
the conditional probabilities

$$
P\left(R_{i} \mid W_{i}, \sigma_{i}\right)
$$

where $\sigma_{i}=S_{i-2} S_{i-1} S_{i} S_{i+1} S_{i+2}$, and $W_{i}$ is the flanking residue window of a width $2 l+1: W_{i}=R_{i-l} R_{i-l+1} \ldots$ $R_{i+l-1} R_{i+l}$. As a convention, we exclude $R_{i}$ from $W_{i}$. Due to the large size 20 of the amino acid alphabet, it is impossible to directly consider residue correlation beyond pair correlation. This means that even for $l=1$ some approximation has to be made. We have proposed a scheme to reduce 20 amino acids into a limited number $(\leq 4)$ of classes for coarse-graining $W_{i}$. Another approximation, which has been also used in the information theory approach GOR, is the approximation of conditional independency. The approximation assumes

$$
\begin{aligned}
P\left(W_{i} \mid \sigma_{i}\right) & =\prod_{j=-l}^{l} P\left(R_{j+i} \mid \sigma_{i}\right), \\
P\left(W_{i} \mid R_{i}, \sigma_{i}\right) & =\prod_{j=-l}^{l} P\left(R_{j+i} \mid R_{i}, \sigma_{i}\right),
\end{aligned}
$$

where the prime indicates that $j=0$ is excluded from the multiplication. The emission probabilities are then reduced to

$$
P\left(R_{i} \mid W_{i}, \sigma_{i}\right)=\frac{P\left(R_{i}, W_{i}, \sigma_{i}\right)}{P\left(W_{i}, \sigma_{i}\right)}=\frac{P\left(R_{i}, \sigma_{i}\right)}{P\left(\sigma_{i}\right)} \frac{\prod_{j}^{\prime} P\left(R_{j+i} \mid R_{i}, \sigma_{i}\right)}{\prod_{j}^{\prime} P\left(R_{j+i} \mid \sigma_{i}\right)} .
$$

Chou-Fasman propensities of amino acid residues for various conformation is a measure of the correlation between a residue and its conformation. The window version of the propensity may written as

$$
\frac{P\left(R_{i}, W_{i}, \sigma_{i}\right)}{P\left(R_{i}, W_{i}\right) P\left(\sigma_{i}\right)} .
$$

The approximation of conditional independency

$$
\begin{aligned}
P\left(W_{i} \mid R_{i}\right) & =\prod_{j=-l}^{l} P\left(R_{j+i} \mid R_{i}\right), \\
P\left(W_{i} \mid R_{i}, \sigma_{i}\right) & =\prod_{j=-l}^{l} P\left(R_{j+i} \mid R_{i}, \sigma_{i}\right),
\end{aligned}
$$

leads expression (6) to

$$
\frac{P\left(R_{i}, \sigma_{i}\right) P\left(W_{i} \mid R_{i}, \sigma_{i}\right)}{P\left(\sigma_{i}\right) P\left(R_{i}\right) P\left(W_{i} \mid R_{i}\right)}=\frac{P\left(R_{i}, \sigma_{i}\right)}{P\left(R_{i}\right) P\left(\sigma_{i}\right)} \frac{\prod_{j}^{\prime} P\left(R_{j+i} \mid R_{i}, \sigma_{i}\right)}{\prod_{j}^{\prime} P\left(R_{j+i} \mid R_{i}\right)},
$$

which consists of the Chou-Fasman propensity and its window correction.

\subsection{Pseudo-counts for probability estimation}

When counts are large, probabilities can be estimated from counts. For example, a unbiased estimate o $P\left(R_{j+i} \mid R_{i}, \sigma_{i}\right)$ from counts is

$$
P\left(R_{j+i} \mid R_{i}, \sigma_{i}\right)=\frac{n\left(R_{j+i}, R_{i}, \sigma_{i}\right)}{n\left(R_{i}, \sigma_{i}\right)}
$$


When counts are small, biased estimations using pseudo-counts are commonly recommended. We use the following background pseudo-count scheme. Assume a background probability distribution $\left\{\rho_{i}\right\}$. From counts $\left\{n_{i}\right\}$ the estimated probabilities $\left\{p_{i}\right\}$ are given by

$$
p_{i}=\frac{n_{i}+\sqrt{n} \rho_{i}}{n+\sqrt{n}}, \quad n=\sum_{i} n_{i}, \quad \sum_{i} \rho_{i}=1 .
$$

In the pseudo-count estimation of $P\left(R_{j+i} \mid R_{i}, \sigma_{i}\right)$, the background distribution is taken to be

$$
\rho\left(R_{j+i} \mid R_{i}, S_{i}\right)=\frac{n\left(R_{j+i}, R_{i}, S_{i}\right)}{n\left(R_{i}, S_{i}\right)}
$$

where, instead of quintuplet $\sigma_{i}$, we look at only the central conformation $S_{i}$. When count $n\left(R_{i}, S_{i}\right)$ vanishes, we simply estimate $P\left(R_{j+i} \mid R_{i}, \sigma_{i}\right)$ to be $\rho\left(R_{j+i} \mid R_{i}, S_{i}\right)$. The background distribution for estimation of $P\left(R_{j+i} \mid R_{i}\right)$ and $P\left(R_{j+i} \mid \sigma_{i}\right)$ are similarly taken to be the simple amino acid frequency $\rho\left(R_{i}\right)$ and $\rho\left(R_{j+i} \mid S_{i}\right)$, respectively. Since $P\left(R_{j+i} \mid R_{i}\right)$ is independent of any structure sequence $S$, no sophisticated estimation would essentially affect the final prediction.

\section{Result}

We create a nonredundant set of 1612 non-membrane proteins for training parameters from PDB_SELECT (Hobohm and Sander, 1994) with amino acid identity less than $25 \%$ issued on 25 September of 2001. The secondary structure for these sequences are taken from DSSP database (Kabsch and Sander, 1983). As mentioned above, the eight states of DSSP are coarse-grained into 3 states: $h, e$ and $c$. This learning set contains 268031 residues with known conformations, among which 94415 are $h, 56510$ are $e$, and 117106 are $c$. There are 296 unknown residues. The size of the learning set is reasonable for training our parameters.

In order to assess the accuracy of our approach, we use the following 2 test sets: Sets rs76 and casp4. A set of 124 nonhomologous proteins is created from the representative database of Rost and Sander (1993) by removing subunits $\mathrm{A}$ and $\mathrm{B}$ of hemagglutinin $3 \mathrm{hmg}$, which are designated as membrane protein by SCOP (Murzin et al, 1995). The 124 sequences and the learning set are not independent of each other according to HSSP database (Dodge, Schneider and Sander, 1998). That is, some proteins of the 124 sequences are homologous with certain proteins in the learning set. Removing the homologous proteins from the 124 sequences and 5 sequences with unknown amino acid segments longer than 6 , we construct Set rs76 of 76 proteins. Nonredundant 34 proteins with known structures of the CASP4 database issued in December of 2000 are taken as Set casp4 (CASP4, 2000).

To assess prediction methods, we calculate for each conformation the sensitivity $s_{n}$ and specificity $s_{p}$

$$
s_{n}=\frac{T P}{T P+F N}, \quad s_{p}=\frac{T P}{T P+F P}
$$

where TP, FP and $F N$ are site counts of the 'true positive', 'false positive' and 'false negative' with respect to the observed real conformation. The total accuracy measure $Q_{3}$ is the total $s_{n}$, i.e. the ratio of the number of correctly predicted sites to the total number of residues to be predicted.

The model using $P\left(R_{i} \mid W_{i}, \sigma_{i}\right)$ for emission probabilities or amino acid scores shows $Q_{3}$ less than $60 \%$. In the following, we consider only the model using the window Chou-Fasman propensities $P\left(R_{i}, W_{i} \mid \sigma_{i}\right) / P\left(R_{i}, W_{i}\right)$ for amino acid scores. It is known that the length distribution of helices deviates from a geometric distribution. The transition rate $T(h h h h h \rightarrow h h h h h)$ may be estimated from the counts of $h h h h h h$ and $h h h h h$ or 
from the average helix length. By using the conditional distribution of helix lengths with lengths not less than $k$, the transition rate is related to the average length $\bar{l}_{h}$ as

$$
T(h h h h h \rightarrow h h h h h)=1-1 /\left(\bar{l}_{h}-k+1\right), \quad \bar{l}_{h}=\sum_{l \geq k} l P(l \mid l \geq k) .
$$

The string counts estimate the transition rate to be 0.88 , while the length distribution with $k=5$ gives 0.82 . They do not coincide. We use an intermediary value 0.83 for the transition rate. For lengths greater than 5 , the approximation of geometric distribution for lengths is good for both $c$ and $e$.

So far we have not fixed the window width. We have examined different window widths from 9 to 19. Results are listed in Table 2. It is seen that the highest accuracy is obtained for width 17. As full probabilistic models, the probability for individual residue to be at various conformation may be calculated as marginal distribution with the so-called Baum-Welch algorithm, besides the inference of structure by searching optimal hidden structure 'path' with the so-called Viterbi algorithm. While the inference from the Baum-Welch marginal posterior is a little superior to that from the Viterbi algorithm in $Q_{3}$ value for test set rs76, it is not the case for casp4.

Besides the popular predictor GOR IV, there is another secondary structure predictor SSP (Solovyev and Salamov, 1991, 1994) based on discriminant analysis using single sequence. To compare with them, their accuracies on the same test sets are also listed in the table.

\section{Discussion}

We have presented simple hidden Markov models to predict secondary structure using single protein sequence. The hidden sequence is generated by a fifth order Markov process, or by the first order Markov process of multi-site conformation states. Considering that structure segments of proteins are generally short, we have ignored the duration effect, and focused on short range correlations.

We combine sliding window scores with hidden Markov models. Two schemes of residue scores investigated are the conditional probability scores and probability ratio scores of propensities. The performance of the latter is much superior to the former. Other score schemes such as GOR scores of information theory may also be used, and should be examined.

The fine classification of windows by quintuplet conformation improves window scores. The HMM integrate scores of different windows, and further reduces prediction error. The $Q_{3}$ accuracy for both viterbi and Baum-Welch algorithms reaches about $69.8 \%$. Since the Baum-Welch algorithm provides posterior distribution over conformation states at each position, the percentage of positions with calculated probability for conformation higher than preset threshold could be taken as a measure of prediction reliability.

We have treated the length distribution simply by changing the transition rate $T(h h h h h \rightarrow h h h h h)$. A more sophisticated way to consider the correction of length distribution is to add only a few new states for long helices up to certain length, say 9 , and then make a geometric distribution approximation for longer helices. The corresponding Markov graph is shown in Fig. 1. The new states and their indices are

68 hhhhh, 69 hhhhh, 70 hhhhhh, 71 hhhhhhh, 72 hhhhhhhh.

States 68 and 69 must follow an $h$, while state 67 follows a non- $h$. State 69 distinguishes from 68 by being exclusively followed by a non- $h$. However, a primary investigation shows the improvement of this correction is insignificant. 
There is scope for further improvement in our approach. We may divide a training set into several, say 2, subsets according to residue statistics. For this purpose, coarse-graining of amino acids would help. The two subsets are then used separately for training to get refined models. We may first classify a query sequence into one of the two categories, and then apply to it the corresponding refined model.

This work was supported in part by the Special Funds for Major National Basic Research Projects and the National Natural Science Foundation of China.

\section{References}

[1] Asai,K., Hayamizu,S., and Handa,K. (1993) Prediction of protein secondary structure by the hidden Markov model, Bioinformatics, 9, 141-146.

[2] CASP4 (2000) http://predictioncenter.llnl.gov/casp4/

[3] Camproux,A.C., Tuffery,P., Chevrolat,J.P., Boisvieux,J.F., Hazout,S. (1999) Hidden Markov model approach for identifying the modular framework of the protein backbone, Protein Eng. 12(12), 10631073.

[4] Chou,P.Y., and Fasman,G.D. (1974a) Conformational parameters for amino acids in helical,betasheet,and random coil regions calculated from proteins. Biochemistry 13(2),211-222.

[5] Chou,P.Y., and Fasman,G.D. (1974b) Prediction of protein conformation.Biochemistry, 13(2), 222-245.

[6] Dodge,C., Schneider,R., and Sander,C. (1998) The HSSP database of protein structure-sequence alignments and family profiles. Nucleic Acids Res, 26, 313-315.

[7] Garnier,J., Gibrat,J.F., and Robson,B. (1996) GOR method for predicting protein secondary structure from amino acid sequence. Methods in Enzymology 266, 540-553.

[8] Garnier,J., Osguthorpe,D., and Robson,B. (1978) Analysis of the accuracy and implications of simple methods for predicting the secondary structure of globular proteins, J. Mol. Biol. 120, 97-120.

[9] Gibrat,J.F.,Garnier,J.,and Robson,B. (1987) Further developments of protein secondary structure prediction using information theory.Newparameters and consideration of residue pairs, J. Mol. Biol. 198, 425-443.

[10] Hobohm,U., and Sander,C. (1994) Enlarged representative set of protein structures, Protein Science 3, $522-524$.

[11] Kabsch,W., and Sander,C. (1983) Dictionary of protein secondary structure: Pattern recognition of hydrogen-boned and geometrical features, Biopolymers 22, 2577-2637.

[12] Karplus,K., Sjolander,K., Barrett,C., Cline,C., Hausser,D., Hughey,R., Holm,L. and Sander,C. (1997) Predicting protein structure using hidden Markov models. Proteins suppl. 1, 134-139.

[13] Murzin,A.G., Brenner,S.E., Hubbard,T., and Chothia,C. (1995) SCOP: A structural classification of proteins database for the investigation of sequences and structures, J. Mol. Biol. 247, 536-540.

[14] Rabiner, L.R. 1989. A tutorial on hidden Markov models and selected applications in speech recognition. Proc. IEEE 77(2), 257-286.

[15] Rost,B., and Sander,C. (1993) Prediction of protein secondary structure at better than $70 \%$ accuracy, J. Mol. Biol. 232, 584-599. 
[16] Schmidler,S.C., Liu,J.S., Brutlag,D.L. (2000) Bayesian segmentation of protein secondary structure, J. Comp. Biol., 7(1/2), pp 233-248.

[17] Solovyev,V.V., and Salamov, A.A. (1991) Method of calculation of discrete secondary structures in globular proteins, Mol. Biol. 25(3), 810-824.

[18] Solovyev,V.V., and Salamov,A.A. (1994) Predicting alpha-helix and beta-strand segments of globular protein, Comput. Appl. Biosci. 10(6), 661-669.

[19] Solovyev,V.V., and Shindyalov,I.N. (2002) Properties and prediction of protein secondary structure, in Current Topics in Computational Molecular Biology, Jiang,T., Xu,Y., and Zhang,M.Q. ed., Tsinghua University Press \& The MIT Press.

[20] Zheng,W.M. (2003) Protein secondary structure prediction based on quintuplets, http://arxiv.org/abs/physics/0307076.

Fig. 1 Markov graph for correction of helix length distribution. Here, the incoming states are 18 chhhh and 42 ehhhh, and outgoing states are $65 h h h h c$ and 66 hhhhe. Other states of multi- $h$ are $67 h h h h h, 68 h h h h h$, 69 hhhhh, 70 hhhhhh, 71 hhhhhhh, and 72 hhhhhhhh. 
Table 1. 68 quintuplet conformation states.

\begin{tabular}{|rc|cc|cc|cc|}
\hline 0 & cccc & 17 & chhhe & 34 & eeech & 51 & heecc \\
1 & ccce & 18 & chhhh & 35 & eeeec & 52 & heeec \\
2 & cccch & 19 & eccc & 36 & eeee & 53 & heeee \\
3 & cccee & 20 & ecce & 37 & eeeeh & 54 & hhccc \\
4 & ccchh & 21 & eccch & 38 & eeehh & 55 & hhcce \\
5 & cceec & 22 & eccee & 39 & eehhh & 56 & hhcch \\
6 & cceee & 23 & ecchh & 40 & ehhhc & 57 & hhcee \\
7 & cceeh & 24 & eceec & 41 & ehhhe & 58 & hhchh \\
8 & cchhh & 25 & eceee & 42 & ehhhh & 59 & hheec \\
9 & ceecc & 26 & echhh & 43 & hcccc & 60 & hheee \\
10 & ceece & 27 & eeccc & 44 & hccce & 61 & hhhcc \\
11 & ceech & 28 & eecce & 45 & hccch & 62 & hhhce \\
12 & ceeec & 29 & eecch & 46 & hccee & 63 & hhhch \\
13 & ceeee & 30 & eecee & 47 & hcchh & 64 & hhhee \\
14 & ceeeh & 31 & eechh & 48 & hceec & 65 & hhhhc \\
15 & ceehh & 32 & eeecc & 49 & hceee & 66 & hhhhe \\
16 & chhhc & 33 & eeece & 50 & hchhh & 67 & hhhhh \\
\hline
\end{tabular}

Table 2. Accuracy of secondary structure predictions for different models. Here, VI and BW stand for 'Viterbi' and 'Baum-Welch' algorithms, respectively. Models discussed are indexed with window widths.

\begin{tabular}{|c|c|rrrrrrr|}
\hline Model & Test set & $S_{n}^{c}$ & $S_{p}^{c}$ & $S_{n}^{e}$ & $S_{p}^{e}$ & $S_{n}^{h}$ & $S_{p}^{h}$ & $Q_{3}$ \\
\hline 9-VI & casp4 & 66.08 & 71.46 & 63.57 & 54.97 & 70.37 & 70.80 & 67.14 \\
9-BW & casp4 & 66.69 & 71.58 & 63.29 & 55.34 & 70.33 & 70.79 & 67.32 \\
9-VI & rs76 & 66.90 & 71.16 & 63.48 & 53.43 & 65.81 & 68.36 & 65.82 \\
9-BW & rs76 & 67.53 & 71.00 & 63.41 & 53.15 & 64.76 & 68.58 & 65.75 \\
11-VI & casp4 & 67.65 & 71.23 & 61.37 & 56.68 & 71.43 & 70.84 & 67.72 \\
15-VI & casp4 & 69.51 & 71.27 & 63.46 & 58.30 & 70.98 & 72.66 & 68.77 \\
17-VI & casp4 & 70.20 & 72.11 & 63.80 & 61.41 & 73.87 & 73.35 & 70.21 \\
19-VI & casp4 & 70.29 & 71.69 & 62.11 & 61.04 & 73.97 & 73.12 & 69.92 \\
17-BW & casp4 & 70.47 & 71.53 & 63.01 & 61.32 & 73.26 & 73.22 & 69.92 \\
17-VI & rs76 & 70.66 & 73.26 & 68.08 & 57.97 & 68.77 & 73.35 & 69.49 \\
17-BW & rs76 & 71.09 & 73.05 & 68.25 & 58.06 & 68.55 & 74.13 & 69.65 \\
\hline GOR4 & rs76 & 79.3 & 66.1 & 54.7 & 55.3 & 63.3 & 68.5 & 66.2 \\
SSP & rs76 & 59.2 & 52.8 & 69.0 & 55.3 & 67.0 & 68.1 & 60.0 \\
GOR4 & casp4 & 81.9 & 62.0 & 43.0 & 54.6 & 67.1 & 64.3 & 63.4 \\
SSP & casp4 & 74.7 & 58.8 & 45.7 & 55.6 & 66.3 & 63.3 & 61.4 \\
\hline
\end{tabular}

